\title{
Signatures of multiple jumps in surface diffusion on honeycomb surfaces
}

\author{
Peter S. M. Townsend* and Nadav Avidor \\ Department of Physics, Cavendish Laboratory, J.J. Thomson Avenue, Cambridge CB3 OHE United Kingdom
}

(Received 19 January 2019; published 13 March 2019)

\begin{abstract}
The jump distribution, a property of the motion of adsorbates on a corrugated surface, contains crucial information on adsorbate-substrate energy dissipation processes. To provide a means to study jump distributions in a honeycomb array of adsorption sites, we derive analytical expressions for the intermediate scattering function (ISF) describing jump diffusion taking into account jumps up to fourth-nearest neighbor in length. To enable testing the analytical expressions against experimental or simulated data, we develop a global fitting routine that can be applied to experimental or simulated ISFs to infer multiple jumps. We demonstrate the analysis method by studying the jump distribution arising from classical Langevin molecular dynamics simulations of two model systems, cyclopentadienyl (Cp) on $\mathrm{Cu}(111)$, and deuterium (D) on $\mathrm{Pd}(111)$. The simulations and analysis confirm that diffusion of $\mathrm{Cp} / \mathrm{Cu}(111)$ at a surface temperature $T_{s}=135 \mathrm{~K}$ takes place in a regime of predominantly single jumps. Classical simulations of $\mathrm{D} / \mathrm{Pd}(111)$ at $T_{s}=350 \mathrm{~K}$, with a realistic Langevin friction, suggest that the diffusion of $\mathrm{D} / \mathrm{Pd}(111)$ involves a high proportion of multiple jumps. The parameters that apply to $\mathrm{D} / \mathrm{Pd}(111)$ are typical of the interaction of hydrogen atoms with close-packed transition metal surfaces, suggesting that long jumps are a general feature of the high temperature surface diffusion of hydrogen.
\end{abstract}

DOI: 10.1103/PhysRevB.99.115419

\section{INTRODUCTION AND MOTIVATION}

An understanding of diffusion-mediated atomic and molecular self-assembly is a leading contribution of fundamental surface science to practical applications of surfaces and interfaces. Relevant areas of application include organic electronics [1] and the synthesis of diverse structures including two-dimensional and nanostructured forms of carbon [2,3], organic porous monolayers [4], and metal nanoclusters [5]. Studies of surface diffusion in model systems contribute by elucidating key qualitative aspects of diffusion including influences of mutual interactions [6,7], rotational motion [8], and quantum tunneling $[9,10]$. Furthermore, the study of model systems allows benchmarking of theoretical treatments of adsorbate-surface interactions, which makes a vital contribution to the ongoing refinement of first-principles techniques for treating adsorption systems [11,12]. Developing more robust theoretical tools in turn is critical to provide reliable insights for complex and applied systems.

Close-packed metal surfaces are a key arena for both the self-assembly applications mentioned above, and also fundamental surface science studies of elementary processes including adsorption [13,14], diffusion [10,15,16], vibration [17], and reaction [18]. On the close-packed face, many atomic and molecular species adsorb preferentially at threefold coordinated hollow sites. A selection of such threefold adsorption systems is collected together in Table I. The close-packed surface presents two symmetrically inequivalent threefold sites, arranged in a so-called honeycomb structure. The honeycomb

\footnotetext{
* Present address: Rutgers University, Department of Chemistry and Chemical Biology, 123 Bevier Road, Piscataway, NJ 08854, USA; peter.townsend@rutgers.edu
}

arrangement will be widely recognized as the atomic structure of some well known two-dimensional materials such as graphene [19] and hexagonal boron nitride [20]. Combined with the preferential adsorption of carbon atoms at hollow sites, the spatial arrangement of hollow sites on close-packed surfaces is therefore a key factor in the prevalent synthesis of graphene on close-packed surfaces [2,21-25]. Diffusion on close-packed surfaces also displays notable features of fundamental interest in surface dynamics. For example, long jumps have been frequently observed in molecular dynamics (MD) simulations of close-packed systems [26-29], despite the curved transition path required for long jumps [30]. We will return to long jumps shortly as a key topic in the present work.

Experimental measurements of surface diffusion at the atomic scale are necessarily constrained in time and energy resolution. Real space imaging methods such as scanning tunneling microscopy (STM) typically operate on the timescale of milliseconds to seconds, providing a window on hopping processes that are particularly slow, either due to low temperatures [15,39] or a large energy barrier to diffusion [6]. By contrast quasielastic atom-surface scattering methods, with resolution in reciprocal space rather than real space, are capable of measuring surface dynamics on picosecond time scales. The experimental focus of the present work is on the helium-3 surface spin echo (HeSE) method [40] which uses magnetic manipulations on a helium-3 beam to enhance the energy exchange resolution in quasielastic scattering, and therefore probe surface dynamics on time scales from subpicosecond up to approximately $1 \mathrm{~ns}$. The interested reader is referred to the review article for further details on the experimental method [40]. Since the HeSE experiment operates in a regime where there is essentially no alternative method, it is important to push the analysis of HeSE data to 
TABLE I. Adsorption systems in which the adsorption sites are the two inequivalent hollow sites on close-packed metal surfaces, to illustrate the wide variety of honeycomb adsorption systems in practice. All the adsorbates shown are atomic except cyclopentadienyl or $\mathrm{Cp}$, which is the five-membered aromatic ring $\mathrm{C}_{5} \mathrm{H}_{5}$. By definition, an fcc site is directly above a substrate atom in the first subsurface layer; the hcp site is the other threefold site. The parameters listed are taken from theoretical studies where possible; values with uncertainties indicate experimental measurements. $V_{b}$ and $\Delta E$ refer to properties of the adiabatic adsorbate-surface potential, either computed with first-principles theoretical methods or constructed to fit experimental data. $V_{b}$ is the diffusion barrier for an adsorbate to escape the lowest energy adsorption site, or the experimental activation energy. $\Delta E$ is the nondegeneracy between the two hollow sites. Quantities that are unknown are indicated by (?).

\begin{tabular}{ccccc}
\hline \hline Adsorbate & Substrate & Stable site & $V_{b}(\mathrm{meV})$ & $\Delta E(\mathrm{meV})$ \\
\hline $\mathrm{H}$ & $\mathrm{Ru}[31]$ & $\mathrm{fcc}$ & 120 & 40 \\
$\mathrm{H}$ & $\mathrm{Pd} \mathrm{[31]}$ & $\mathrm{fcc}$ & 125 & 25 \\
$\mathrm{C}$ & $\mathrm{Ru}[32]$ & $\mathrm{fcc}$ & 870 & 740 \\
$\mathrm{C}$ & $\mathrm{Ni}[3,33,34]$ & $\mathrm{hcp}$ & $400-480$ & 40 \\
$\mathrm{O}$ & $\mathrm{Ru}[35]$ & $\mathrm{hcp}$ & 700 & 400 \\
$\mathrm{Na}$ & $\mathrm{Cu}[16,36]$ & $(?)$ & $12 \pm 3$ & $(?)$ \\
$\mathrm{Cp}$ & $\mathrm{Cu}[37,38]$ & $(?)$ & $41 \pm 1$ & $10.6 \pm 1.7$ \\
\hline \hline
\end{tabular}

its limits to maximize the information that can be gained from the technique. In our present study we build upon a powerful and sensitive method for analyzing jump diffusion on a closepacked surface $[10,38]$. We apply the method to the output of molecular dynamics simulations, establishing the potential for application to experimental data in the near future.

Long jumps have frequently been inferred from MD simulation by direct inspection of trajectories, along with a suitable definition of what constitutes a jump $[28,29,41]$. However, the HeSE technique is a reciprocal space method requiring a different analysis approach, in which the key concept is the intermediate scattering function (ISF). The ISF is routinely computed from MD simulations in order to place simulation and experiment on the same footing when analyzing HeSE data $[16,37,42]$, and we follow the same approach in the present work. The ISF is the Fourier transform in space of van Hove's conditional probability function [43] conventionally called $G(\mathbf{R}, t)$. Classically, $G(\mathbf{R}, t)$ gives the conditional probability of finding a particle at position $\mathbf{R}^{\prime}+\mathbf{R}$ at time $t^{\prime}+t$ given that there was a particle at position $\mathbf{R}^{\prime}$ at time $t^{\prime}$, integrated over all $\mathbf{R}^{\prime}, t^{\prime}$. $G(\mathbf{R}, t)$ therefore gives a powerful and general statistical description of the equilibrium dynamics of a set of scattering centers. The ISF $I(\Delta \mathbf{K}, t)$ represents the same detailed information except in a spatial Fourier domain where $\Delta \mathbf{K}$ is the variable conjugate to $\mathbf{R}$. The ISF is very closely related to the raw data measured in HeSE experiments [42], and hence by developing our analysis framework in terms of the ISF, the resulting tools can be applied to analyze HeSE data.

A key part of the analysis toolbox for HeSE experiments is the existence of simple analytical forms for the ISF corresponding to different qualitative types of adsorbate dynamics, including ballistic motion, continuous diffusion, and jump diffusion [40]. The relatively simple case where hopping takes place over a Bravais lattice of adsorption sites is covered by the Chudley-Elliott model [44], and has been shown to apply in a number of HeSE experiments including diffusion on close-packed surfaces where adsorption occurs on top sites [7,45]. In the Chudley-Elliott model, the ISF is rigorously described at fixed $\Delta \mathbf{K}$ by a monoexponential decay in $t$. The decay rate, conventionally called $\alpha(\Delta \mathbf{K})$, is a sinusoidal function of $\Delta \mathbf{K}$ which depends on the structure of the surface and also on the relative rates of nearest-neighbor (single) jumps, second-nearest-neighbor (double), and higher order jumps. In Chudley-Elliott systems, the inference of a jump distribution from either simulation or experiment is in principle relatively straightforward, in the absence of complicating factors such as strong mutual interactions. Broadly speaking, the $\alpha(\Delta \mathbf{K})$ can be obtained by least-squares fitting individual ISFs, and the $\alpha(\Delta \mathbf{K})$ itself can then be analyzed to yield information about the jump distribution [46]. The Chudley-Elliott model does not apply to diffusion on honeycomb surfaces, since not all adsorption sites are equivalent. General expressions for the ISF in non-Bravais systems have been worked out previously $[47,48]$, but their application to experimental data has been limited to systems in which multiple jumps are negligible $[37,38]$. The analytical details are recapped and extended in Sec. II. From the point of view of data analysis, the most notable feature of the analytical results for non-Bravais systems is their multiexponential form. Multiexponential functions are famously difficult to fit reliably [49], which either drastically increases the signal to noise requirements of the experiment, or demands a more sophisticated data analysis approach. A method with increased statistical power, based on the Bayesian probability-based concept of data fitting, has previously been described and applied to analyze diffusion in the honeycomb systems $\mathrm{H} / \mathrm{Ru}(0001)$ [10] and $\mathrm{Cp} / \mathrm{Cu}(111)$ [38]. However, the possibility of multiple jumps was neglected, and incorporating multiple jumps into the analysis is a critical step in studying systems where a low friction regime is plausible, in order to arrive at a physically consistent interpretation of the dynamics.

To that end, we present a framework for inferring the presence of long jumps in diffusion on honeycomb surfaces, where the statistical description of the motion is expressed via the ISF. We demonstrate the relevance of the method to physical systems, by studying the Langevin dynamics of two model surface systems. In Sec. II we extend a previous derivation of the ISF for non-Bravais lattice adsorption systems, to include long jumps up to fourth-nearest neighbor in length. In Sec. III we give details of the numerical simulation and analysis algorithms used in the study, namely Langevin MD simulations, calculation of the ISF from those simulations, and the marginalized global fitting method. We study the classical Langevin dynamics of the model systems $\mathrm{Cp} / \mathrm{Cu}(111)$ and $\mathrm{D} / \mathrm{Pd}(111)$ and explore the effect of friction on long jumps in a physically realistic parameter range.

\section{THEORY}

The unifying object of study in the present work is the ISF, which connects MD simulations, hopping models, and the HeSE experiment. In the Introduction, the ISF $I(\Delta \mathbf{K}, t)$ was defined as the spatial Fourier transform of a conditional 
probability function $G(\mathbf{R}, t)$. However, $I(\Delta \mathbf{K}, t)$ can be given an equivalent definition that allows a direct calculation from MD or MC dynamics simulations. For a single particle moving in two dimensions with time-dependent position $\mathbf{R}(t)$, the ISF can be written as

$$
I(\Delta \mathbf{K}, t)=\left\langle e^{i \mathbf{K} \cdot \mathbf{R}(t)} e^{-i \Delta \mathbf{K} \cdot \mathbf{R}(0)}\right\rangle,
$$

where angle brackets represent a time average. Additionally, we perform several independent simulations, representing an ensemble of noninteracting adsorbates, and average the result over all the trajectories. For uncorrelated motion of independent adsorbates, such a procedure is equivalent to the more general definition of the ISF for a classical manyparticle system [50]. At fixed $\Delta \mathbf{K}$ the ISF is a function of a correlation time $t$, and the decay of the ISF represents a loss of correlation as a result of aperiodic processes, including random hopping but also the continuous aperiodic motion within each adsorption site known as intracell diffusion or vibrational dephasing [40,51]. The parameter $\Delta \mathbf{K}$ is both the Fourier variable conjugate to $\mathbf{R}$, and the mean quasielastic surface-parallel momentum transfer when the ISF is measured in a HeSE experiment [40].

The ISF has previously been derived for jump systems with multiple adsorption sites per unit cell $[47,48]$, of which honeycomb surfaces are a special case. The ISF for a particle hopping on a surface with two adsorption sites per unit cell takes the form of a biexponential decay, i.e.,

$$
I(\Delta \mathbf{K}, t)=A_{1}(\Delta \mathbf{K}) e^{-\alpha_{1}(\Delta \mathbf{K}) t}+A_{2}(\Delta \mathbf{K}) e^{-\alpha_{2}(\Delta \mathbf{K}) t},
$$

where $A_{1,2}$ and $\alpha_{1,2}$ are known as the amplitudes and decay rates. The analytical solution for $A_{1,2}(\Delta \mathbf{K})$ and $\alpha_{1,2}(\Delta \mathbf{K})$ can be found by solving a master equation for the evolution of the probability over the adsorption sites [48], assuming that jumps from any given site are independent Poisson processes. We assume further that, once multiple jumps are included in the jump distribution, the jump process is Markovian. Instead of working directly from the master equation, we begin our main derivation starting from the recipe set down in previous work [48].

The honeycomb structure consists of two interleaved sublattices, labeled 1 and 2 . We write the jump rate from sublattice $\mu$ to $v$ via jump vectors $\mathbf{l}_{\mu \nu k}$ as $1 / \tau_{\mu \nu k}$, where $k$ is an index running over all allowable jumps connecting sublattices $\mu$ and $v$. Define a $2 \times 2$ matrix $\mathbf{A}$ with elements

$$
A_{\mu \nu}=\sum_{k} \frac{1}{\tau_{\nu \mu k}} \exp \left(-i \Delta \mathbf{K} \cdot \mathbf{l}_{\mu \nu k}\right)-\delta_{\mu \nu} \sum_{v^{\prime}} \frac{1}{\tau_{\mu v^{\prime}}},
$$

where $1 / \tau_{\mu \nu}$ is the total jump rate from sublattice $\mu$ to $\nu$. Let $c_{\mu}$ be the relative equilibrium concentration of particles in sublattice $\mu . c_{1}$ and $c_{2}$ are related by a constant $\lambda$ defined by $c_{2}=\lambda c_{1}$. The absolute values of $c_{1}$ and $c_{2}$ do not affect the final answer; only their ratio is important. Detailed balance implies that any pair of hopping rates $1 \rightarrow 2$ and $2 \rightarrow 1$ are also in the ratio $\lambda$. We define a matrix $\mathbf{T}$ with elements

$$
T_{\mu \nu}=\frac{1}{c_{\mu}} \delta_{\mu \nu},
$$

and construct the (Hermitian) matrix $\mathbf{B}$,

$$
\mathbf{B}=\mathbf{T A T}^{-1},
$$

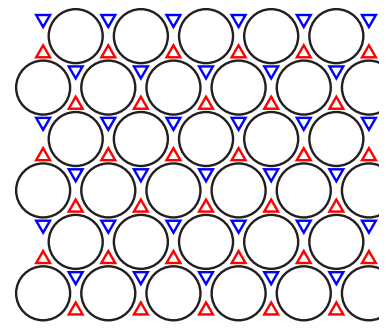

(a) Threefold adsorption sit among surface atoms.

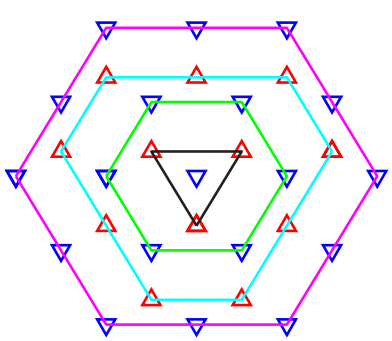

(b) Different classes of jump represented by shells.
FIG. 1. Honeycomb arrangement of adsorption sites, and jumps between the sites. (a) The arrangement of threefold adsorption sites among the substrate atoms of a close-packed surface such as $\mathrm{Cu}(111)$ or Pd(111). Substrate atoms are shown as unfilled circles, while upward pointing (blue) and downward pointing (red) triangles show the threefold adsorption sites. The two classes of adsorption site form two inequivalent sublattices. (b) The different types of jump available from a selected site. First through fourth order jumps are represented by shells showing the available target sites. The black triangle has as its vertices the final sites reachable by single jumps. The green regular hexagon plays the same role for double jumps, and the magenta regular hexagon for quadruple jumps. The target sites associated with triple jumps, as defined in the present article, form an irregular hexagon with threefold symmetry rather than sixfold.

with eigenvalues $-\alpha_{p}(p=\{1,2\})$ and the associated normalized eigenvectors $\mathbf{b}^{p}$, whose elements will be called $b_{\mu}^{p}$. Defining the decay amplitudes as

$$
A_{p}(\Delta \mathbf{K})=\left|\sum_{\mu} \sqrt{c_{\mu}} b_{\mu}^{p}\right|^{2},
$$

the ISF is given by

$$
I(\Delta \mathbf{K}, t)=\sum_{p} A_{p}(\Delta \mathbf{K}) \exp \left[-\alpha_{p}(\Delta \mathbf{K}) t\right] .
$$

The result so far is valid for arbitrary long jumps, but previously explicit expressions were given for single jump processes only. The rates associated with different types of jump on the surface enter the derivation at the stage where $\mathbf{A}$ is constructed. Next we give the associated jump vectors and use them to construct $\mathbf{A}$ considering jumps of up to fourth-nearest neighbor in length.

\section{A. Jump vectors up to fourth order}

We refer to nearest neighbor, next-nearest neighbor, thirdnearest neighbor, and fourth-nearest neighbor jumps as single, double, triple, and quadruple jumps, respectively. The arrangement of adsorption sites, and the jumps between them, are represented in Fig. 1. Single jumps connect inequivalent sites. For each site there are three possible single jumps. We define sites of type $\mu=1$ as those where one of the outbound single jumps is in the negative $y$ direction. We define $a$ as the close-packing distance, the separation of nearestneighbor surface atoms. We will describe all jump vectors in a Cartesian basis where the first basis vector ( $\mathbf{x}$, say) points horizontally to the right in Fig. 1, and the second basis vector $(\mathbf{y})$ points in the positive $y$ direction. By inspection of 
Fig. 1, the available single jump vectors from sublattice 1 are $a\left(0,-\frac{1}{\sqrt{3}}\right), a\left(\frac{1}{2}, \frac{1}{2 \sqrt{3}}\right)$, and $a\left(-\frac{1}{2}, \frac{1}{2 \sqrt{3}}\right)$. The destinations available via single jumps, from a selected adsorption site, are indicated by the black triangle in Fig. 1(b). The available single jumps from sites of type 2 are their negatives. Double jumps are between equivalent sites, and are indicated by the green hexagonal shell in Fig. 1, the smaller of the two regular hexagons in the figure. For sites of either hollow type, there are six available double jumps and the jump vector sets are identical for both types of site. In full, they are $\pm a(1,0)$, $\pm a\left(\frac{1}{2}, \frac{\sqrt{3}}{2}\right)$, and $\pm a\left(\frac{1}{2},-\frac{\sqrt{3}}{2}\right)$.

There exist two different lengths of triple jump, whose target sites form the irregular cyan hexagon in Fig. 1. We will follow previous work [52] and assume that the two distinct types of triple jump occur at equal rates, on the basis that the corresponding minimum-energy path (MEP) in the continuous potential is very similar between the two cases: the MEP possesses three approximately straight barrier-crossing sections connected by wells (the hollow sites). The three shorter triple jump vectors are to those target sites forming the midpoints of the long sides of the irregular hexagon, and are given by $a\left(0, \frac{2}{\sqrt{3}}\right)$ and its two further threefold $\left(120^{\circ}\right)$ rotations. The six longer triple jump vectors, to the target sites forming the vertices of the irregular hexagon, are given by $a\left(\frac{3}{2}, \frac{1}{2 \sqrt{3}}\right)$ and its threefold rotations, and $a\left(1, \frac{2}{\sqrt{3}}\right)$ and its threefold rotations.

Finally, quadruple jumps connect equivalent sites. Each site in the honeycomb has 12 fourth-nearest-neighbor sites, defined by being connected to the current site by four successive single jumps and not having a more direct jump path available. The target sites from the selected site in Fig. 1 form the magenta regular hexagon, the larger of the two regular hexagons in the figure. The associated jump vectors are $a(2,0), a\left(\frac{3}{2}, \frac{\sqrt{3}}{2}\right), a(1, \sqrt{3})$, and $a(0, \sqrt{3})$, with all pairs of \pm allowed for both components. Six of the jump vectors, for example those in the $y$ direction, can be achieved by two different paths, giving 18 types of quadruple jump in total.

\section{B. Construction of the matrix A}

It is convenient to write the total jump rate out of sublattice $\mu=1$ as $\tau^{-1}$, which defines $\tau$ as a mean residence time. Then we divide the jumps up into single (s), double (d), triple $(\mathrm{t})$, and quadruple (q) jumps in the proportions $p_{1}, p_{2}, p_{3}$, and $p_{4}$, respectively, such that the total jump rates out of sublattice $\mu=1$ due to $\mathrm{s}, \mathrm{d}, \mathrm{t}$, and $\mathrm{q}$ jumps are given by $p_{1} / \tau, p_{2} / \tau, p_{3} / \tau$, and $p_{4} / \tau$, respectively. The rates associated with jumps along any specific jump vector are found by dividing the total $\mathrm{s} / \mathrm{d} / \mathrm{t} / \mathrm{q}$ jump rate by the number of jumps of that class. For example, the rate of single jumps in the negative $y$ direction out of $\mu=1$ sites is $p_{1} /(3 \tau)$. The third and fourth order neighbors can both be divided up into two subclasses, differing in their straight-line distance to the initial site. In each case, the sites further away from the initial site are those on the vertices of the hexagons in Fig. 1, and are accessible by only one route with the given number of intermediate sites. Those sites on the edges rather than the vertices of the cyan (magenta) hexagon are accessible via two distinct but equivalent triple (quadruple) jumps. We account for the multiple routes by counting those jump vectors twice [52]. The number of distinct types of triple (quadruple) jump is therefore 12 (18), including repeated jump vectors, even though the number of target sites is 9 (12).

The matrix elements of $\mathbf{A}$ along an arbitrary direction cannot be written down explicitly and concisely. As in previous studies [38,48], we instead evaluate $\mathbf{A}$ along the high symmetry directions of the surface, namely the $\mathbf{x}$ direction and the $\mathbf{y}$ direction. The restriction to specific high symmetry surface directions could be lifted easily in principle, but the expressions would be cumbersome to write down, and would not reflect the typical data acquisition routine in experiments.

The matrix elements of $\mathbf{A}$, when $\Delta \mathbf{K}$ is parallel to $\mathbf{x}$, can be written down by evaluating the general prescription (3) and using the jumps as described above. The top left diagonal element of $\mathbf{A}$ contains contributions only from jumps between equivalent sites, which is a double jump contribution $A_{11}^{(d)}$ and a quadruple jump contribution $A_{11}^{(q)}$. Additionally, the diagonal elements of $\mathbf{A}$ contain the $\Delta \mathbf{K}$-independent term $\sum_{\mu^{\prime} \nu^{\prime}} \tau_{\mu^{\prime} \nu^{\prime}}^{-1}$ which, using the definition introduced for $\tau$, is simply $\frac{1}{\tau}$ for the $A_{11}$ element. In total,

$$
\begin{gathered}
A_{11}=A_{11}^{(d)}+A_{11}^{(q)}-\frac{1}{\tau}, \\
A_{11}^{(d)}=\frac{p_{2}}{6 \tau}\left[2 \cos (\Delta K a)+4 \cos \left(\frac{\Delta K a}{2}\right)\right], \\
A_{11}^{(q)}=\frac{p_{4}}{18 \tau}\left[4+4 \cos (\Delta K a)+8 \cos \left(\frac{3 \Delta K a}{2}\right)\right. \\
+2 \cos (2 \Delta K a)] .
\end{gathered}
$$

The second diagonal matrix element $A_{22}$ can be plausibly related to $A_{11}$ by assuming that the jump proportions for jumps out of type 1 sites and type 2 sites are the same. Under that assumption, and by appealing to detailed balance [53] between the two populations of adsorption sites such that, for example $\tau_{12}=\lambda \tau_{21}$, we can write

$$
A_{22}=A_{11} / \lambda \text {. }
$$

If there was a very strong degeneracy between the two sites, such that trapping of a particle was far more likely in the deeper of the two adsorption wells, then assumption (11) could lead to significant problems with the model. However, the practical utility of the model is for systems where $\lambda$ is of order unity, the site energy difference is much less than the diffusion barrier, and the probability of a particle to be trapped when passing through either type of site is not much different. Therefore, we make the assumption (11) throughout the present work, but note that there are circumstances where relaxing the assumption may be beneficial.

The top-right off-diagonal element $A_{12}$ of $\mathbf{A}$ can be broken down into a single jumps contribution $A_{12}^{(s)}$ and a triple jumps contribution $A_{12}^{(t)}$ :

$$
\begin{gathered}
A_{12}=A_{12}^{(s)}+A_{12}^{(t)}, \\
A_{12}^{(s)}=\frac{p_{1}}{3 \tau \lambda}\left[1+2 \cos \left(\frac{\Delta K a}{2}\right)\right],
\end{gathered}
$$




$$
\begin{aligned}
A_{12}^{(t)}= & \frac{p_{3}}{6 \tau \lambda}[1+2 \cos (\Delta K a)]+\frac{p_{3}}{12 \tau \lambda}\left[2 \cos \left(\frac{3 \Delta K a}{2}\right)\right. \\
& \left.+2 \cos (\Delta K a)+2 \cos \left(\frac{\Delta K a}{2}\right)\right]
\end{aligned}
$$

The remaining off-diagonal element $A_{21}$ is related to $A_{12}$ by detailed balance,

$$
A_{21}=\lambda A_{12}^{*}
$$

When $\Delta \mathbf{K}$ is parallel to $\mathbf{y}$, vertical on the page in Fig. 1, the matrix elements of $\mathbf{A}$ are constructed in the same way as just presented, except for inserting the $y$ components of the jump vectors instead of the $x$ components. The diagonal elements are again written in terms of a double jump rate, quadruple jump rate, and total exit rate, and the results read

$$
\begin{gathered}
A_{11}=A_{11}^{(d)}+A_{11}^{(q)}-\frac{1}{\tau} \\
A_{11}^{d}=\frac{p_{2}}{6 \tau}\left[2+4 \cos \left(\frac{\sqrt{3}}{2} \Delta K a\right)\right] \\
A_{11}^{(q)}=\frac{p_{4}}{18 \tau}\left[2+8 \cos \left(\frac{\sqrt{3}}{2} \Delta K a\right)+8 \cos (\sqrt{3} \Delta K a)\right]
\end{gathered}
$$

$$
A_{22}=A_{11} / \lambda \text {. }
$$

The off-diagonal elements are written as the sum of a single jumps and triple jumps contribution:

$$
\begin{gathered}
A_{12}=A_{12}^{(s)}+A_{12}^{(t)}, \\
A_{12}^{(s)}=\frac{p_{1}}{3 \tau \lambda}\left[2 \exp \left(\frac{-\sqrt{3}}{2} i \Delta K a\right)\right] \\
A_{12}^{(t)}=\frac{p_{3}}{12 \tau \lambda}\left[2 \exp \left(-\frac{2 i \Delta K a}{\sqrt{3}}\right)+4 \exp \left(\frac{i \Delta K a}{\sqrt{3}}\right)\right] \\
+\frac{p_{3}}{12 \tau \lambda}\left[2 \exp \left(-\frac{i \Delta K a}{2 \sqrt{3}}\right)+2 \exp \left(-\frac{2 i \Delta K a}{\sqrt{3}}\right)\right. \\
\left.+2 \exp \left(\frac{5 i \Delta K a}{2 \sqrt{3}}\right)\right] . \\
A_{21}=\lambda A_{12}^{*} .
\end{gathered}
$$

The matrix $\mathbf{A}$ can then be processed into the ISF as prescribed by Eqs. (4) to (7). In principle, explicit closed forms for the ISF could be written down, as done for the model including only single jumps [48], since the diagonalization of a $2 \times 2$ matrix can be carried out in closed form. However, the resulting expressions would be significantly more cumbersome than the individual elements of $\mathbf{A}$, and so we leave the analytical working in the form of an exact expression for $\mathbf{A}$. In practice, in the implementation accompanying the present paper, the diagonalization of the matrix $\mathbf{B}$ is performed using closed form expressions for the eigenvectors and eigenvalues of a $2 \times 2$ matrix.

\section{Survival probability model}

Although the expressions developed so far for $I(\Delta \mathbf{K}, t)$ are completely general with respect to the proportions $p_{1}$ through $p_{4}$, it is reasonable to apply a constraint to the proportions, both on physical grounds and in order to reduce the number of free parameters of the model. Here we assume a survival probability model [52], in which the rate of individual single, double, triple, and quadruple jumps are in a geometric sequence $1: s: s^{2}: s^{3}$. There is no fundamental reason why the jump rates must be related exactly in that way, and so the introduction of $s$ rather than three independent jump rates is an approximation, but one that allows us to explore the prevalence of long jumps via a single parameter. The net rates of all single, all double, all triple, and all quadruple jumps are then in the ratio $p_{1}: p_{2}: p_{3}: p_{4}=3: 6 s: 12 s^{2}: 18 s^{3}$. The absolute proportions $p_{1}$ are determined by the normalization $p_{1}+p_{2}+p_{3}+p_{4}=1$.

With a definite model for the proportions of the rates of different lengths of jump, the analytical theory of the present section can be confirmed by carrying out kinetic Monte Carlo (KMC) simulations of the jump motion. The confirmatory results of KMC calculations, and further background on the KMC method [10,54-58], are provided in the Supplemental Material [59].

\section{Separation of timescales}

To interpret continuous classical MD simulations in terms of jump motion, we need to distinguish jumps from shorttimescale dynamics such as ballistic and frustrated translational motion. A well-defined separation of vibrational and hopping timescales is not guaranteed for motion in a general potential, but is compulsory in order to have a well-defined jump rate [60]. The systems we study in the present work possess sufficiently strongly corrugated potentials such that the vibrational and jump motion are cleanly separated, on a timescale of the inverse friction $1 / \gamma$, as clearly seen later in Fig. 5. The analysis of all ISFs in the present work therefore begins by retaining only the long-time tail of the correlation function which contains information about jump motion rather than intracell processes.

The long-time tail can be defined by introducing a cut-off correlation time for each ISF, corresponding to a timescale which delineates the fast intracell processes, and slower jump processes, represented in the correlation function. The use of a cut-off time is meaningful as long the results are insensitive to the exact choice of the cutoff. In other words if the final outputs of the analysis converge with respect to the cut-off time then we can conclude that the ISF for times longer than the parameters extracted from the tails of the ISF have a meaningful interpretation in terms of a coarse-grained description of the dynamics as a jump process. For both model systems presented later on, the jump rates and survival probabilities extracted by the global analysis routine typically vary by a fraction of a percent if the cut-off time is varied by a factor of 5 from $1 / \gamma$ to $5 / \gamma$.

The use of $1 / \gamma$ as the default timescale of the cutoff is expected based on a previous comparison of jump rates measured by analysis of ISFs compared to trajectory inspection in a further hexagonal system [Xe/Ni(111)] displaying multiple 
jumps [61]. In our case of $\mathrm{D} / \mathrm{Pd}(111)$, use of $1 / \gamma$ as the cut-off time leads to a small but significant drift in the fitted value of $\lambda$ the concentration ratio in the jump model, as a function of $\gamma$. However, when the cut-off time is increased to $3 / \lambda$ there is no systematic drift in $\lambda$, showing that the underlying jump model is consistent with the simulated dynamics at a range of $\gamma$, as long as the cut-off time is chosen sufficiently long. By contrast, for $\mathrm{Cp} / \mathrm{Cu}(111)$ choosing a cut-off time of $1 / \gamma$ does not lead to any spurious drift in $\lambda$. Together the results suggest that $1 / \gamma$ provides an approximate minimum cut-off time, but may be a little too short to obtain properly converged measures of the jump process, depending on the other system parameters.

\section{NUMERICAL METHODS}

\section{A. Langevin molecular dynamics}

In the present work the Langevin equation (LE) is used as a simple and largely physical model of fluctuation and dissipation in surface dynamics. We use the Verlet algorithm [62] to simulate the LE, which reads [50]

$$
m \ddot{\mathbf{R}}=-\nabla V(\mathbf{R})-m \gamma \dot{\mathbf{R}}+\mathbf{F}(t),
$$

where $m$ is the adsorbate mass, $V(\mathbf{R})$ is the adiabatic potential energy surface (PES) or potential of mean force, $\gamma$ is the dynamical friction (viscosity per unit mass) with units of frequency, and $\mathbf{F}(t)$ is a random force following a Gaussian distribution with zero mean. Assuming the friction acts isotropically, the autocorrelation of $\mathbf{F}$ is given by the standard classical fluctuation-dissipation relation [63] $\left\langle F_{i}(t) F_{j}(0)\right\rangle=$ $2 m k_{B} T \delta(t) \delta_{i, j}$ with $\delta(t)$ the Dirac delta function and $\delta_{i, j}$ a Kronecker- $\delta$ symbol over the two Cartesian spatial directions of the simulation. Our simulations are run with a fixed time step $\Delta t$, and the random force is constructed to have the autocorrelation

$$
\left\langle F_{i}(t) F_{j}(0)\right\rangle=\frac{2 m k_{B} T}{\Delta t} \delta_{i, j} .
$$

The LE maintains the simulation at temperature $T$ and provides a single adjustable parameter to represent the strength of dissipative coupling between the adsorbate and the sources and sinks of energy present on the surface, including electronhole pair excitations and surface phonons [30].

The potential energy surface $V(\mathbf{R})$ is implemented by tabulating the associated force $-\nabla V(\mathbf{R})$ on a very fine grid within a primitive unit cell before the simulation begins, and applying a wrap-and-lookup operation at every time step. For potentials described by a simple Fourier series, the lookup method can give a minor speed advantage with negligible loss of accuracy. However, not all atom/surface interaction potentials are well described by a low-order Fourier series, and instead lend themselves to alternative representations such as spline interpolation between samples on a grid. In the two MD simulations of the present work, one potential energy surface was constructed analytically, and one was constructed by interpolation of numerical data from the literature. The ability to treat both simple Fourier-series potentials and numerically interpolated potentials on the same footing, combined with the speed advantage, is the motivation for using the lookup method. The grid resolution in the present work was approximately $1 \times 10^{-3} \AA$, at which level the approximation associated with the finite resolution and grid lookup has no discernible influence on the results.

\section{B. Construction of the PES}

We describe the construction of the two-dimensional potential energy surface $V(\mathbf{R})$ for the Langevin molecular dynamics simulations of $\mathrm{D} / \mathrm{Pd}(111)$ and $\mathrm{Cp} / \mathrm{Cu}(111)$ described in Sec. IV. For $\mathrm{D} / \mathrm{Pd}(111)$, numerical values of $V(\mathbf{R})$ on a Cartesian grid were obtained from a density functional theory (DFT) study of several hydrogen/metal systems [31]. The data were mapped by symmetry operations onto a rectangular (nonprimitive) surface unit cell containing two primitive unit cells, and interpolated onto a fine grid of $400 \times 700$ points using the MATLAB function csape [64] with periodic boundary conditions. The interpolation to a finer $2000 \times 3500$ grid, to allow an accurate evaluation of the force by finite differences, was then performed with interp2 [65]. The resulting potential energy surface is smooth and free of apparent artifacts. The two-dimensional potential, and a one-dimensional slice representing the diffusion pathway between hollow sites over bridge sites, are shown in Fig. 2.

Although some first-principles data is available for the $\mathrm{Cp} / \mathrm{Cu}(111)$ system [66], there is not enough to repeat the method used to construct the D/Pd(111) PES from DFT data. Instead, the $\mathrm{Cp} / \mathrm{Cu}(111)$ potential was constructed by adding smooth localized functions to a low order Fourier series, in a way that achieves consistency with prior experimental data. The method is described in further detail in the Supplemental Material $[16,37,38,45,59,67]$. Despite the different construction route, the $\mathrm{Cp} / \mathrm{Cu}(111)$ PES is qualitatively similar to the D/Pd(111) PES shown in Fig. 2. The analogous plot of the $\mathrm{Cp} / \mathrm{Cu}(111)$ potential is provided in the Supplemental Material.

\section{Numerical intermediate scattering function}

The ISF for a single simulated trajectory $\mathbf{R}(t)$ is calculated using Eq. (1). The time average is computed efficiently using the (discrete) Wiener-Khinchine theorem [68]. The incoherent average over independent trajectories was performed after the inverse Fourier transform so that as well as the mean of the ISF, the variance of the ISF can also be recorded directly. The variance of the ISF at each point in time is required input for the global fitting method, to be described shortly.

A zero-padding scheme was used to avoid the spurious measurement correlations between the two ends of the nonperiodic trajectories. However, for the analysis of ISFs within the jump model, only 5\% of the full time range of the ISF was retained, making the difference between the two methods minimal. For example, the simulations of $\mathrm{D} / \mathrm{Pd}(111)$ were run up to a simulation time of $1.2 \mathrm{~ns}$ but only points in the ISF up to $60 \mathrm{ps}$ were retained for analysis.

\section{Global marginalized fitting}

A key aspect of the analysis in the current study is the use of a global fitting method. If the adsorption sites formed a Bravais lattice, then the jump dynamics could be extracted by the standard method of fitting $I(\Delta \mathbf{K}, t)$ to an exponentially decaying function of $t$ with decay rate $\alpha(\Delta \mathbf{K})$, and comparing 


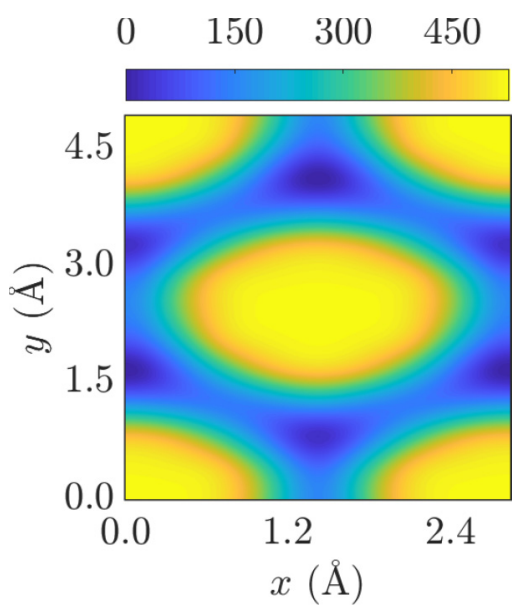

(a)

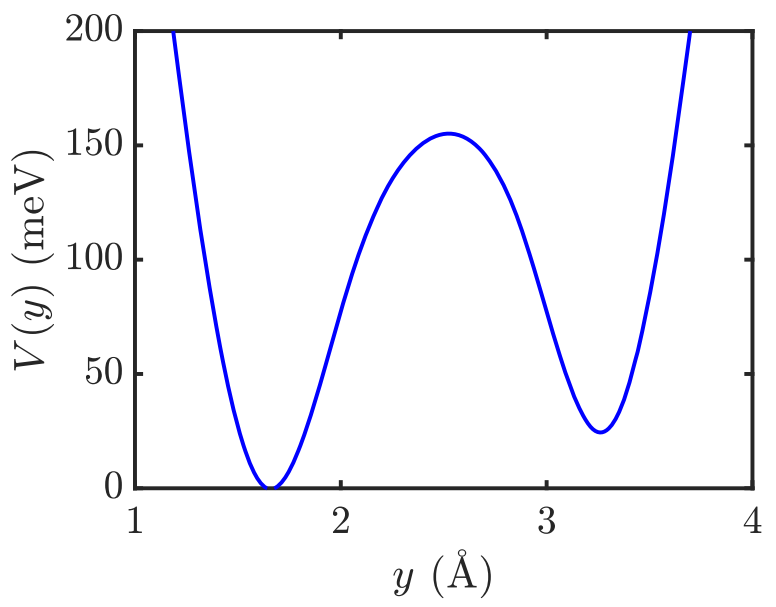

(b)

FIG. 2. (a) The two-dimensional potential energy surface for $\mathrm{D} / \mathrm{Pd}(111)$ interpolated from numerical DFT data as described in the main text. The scale bar above the plot shows the potential in meV. The bright yellow maxima are top sites, which are all equivalent. The dark blue minima are hollow sites. There are two nondegenerate hollow sites per primitive unit cell. (b) A one-dimensional cross section at $x=0$ showing the energy barrier to diffusion between neighboring hollow sites. The nondegeneracy $\Delta E \approx 25 \mathrm{meV}$ of the neighboring hollows is more clearly visible in the one-dimensional plot. The diffusion barrier is seen to be approximately $150 \mathrm{meV}$ from the lower-energy hollow.

$\alpha(\Delta \mathbf{K})$ to the analytical form of Chudley and Elliott [44]. However, that model does not apply to our honeycomb surface where in general the ISF at fixed $\Delta \mathbf{K}$ consists of a biexponential decay. Each biexponential is difficult to fit directly unless given exceptionally favorable underlying parameters and a very high signal-to-noise ratio. Therefore, we focus here on establishing a robust global analysis method based on analyzing the ISF over all available $\Delta \mathbf{K}$.

The core of our global fitting method is a function $B$ that returns the probability of obtaining a set of values $\left\{I_{i}\right\}$ for the ISF at fixed $\Delta K$ given a set of correlation times $\left\{t_{i}\right\}$, corresponding uncertainties $\left\{\sigma_{i}\right\}$, and a model function $f(\Delta K, t)$ for the normalized ISF. In the present investigation, the function $f(\Delta K, t)$ depends on the model parameters $\tau, \lambda$, and $s$. Therefore for a given simulation data set the function $B$ can be summarized as a function of the three model parameters $B(\tau, \lambda, s)$. The probability is marginalized over linear combinations of the model function and a static level $(u f+$ $v$ ), for physical reasons as discussed in the Supplemental Material [59], where we write down an explicit expression for $B$ based on previous formulations $[38,69]$.

By Bayes' theorem [38,49], $B$ is proportional to the posterior probability of $(\tau, \lambda, s)$ given the data set and model, under the assumptions of independent normally distributed uncertainties, uniform prior probability distributions in $\tau, \lambda$, and $s$, and that the choice of integration limits for marginalization as $\pm \infty$ rather than their physical bounds of 0 to 1 makes no significant difference to the result. In edge cases of extremely noisy data or ISFs that decay very slowly compared to the time window of the simulation or experiment, the integration limits may need to be considered more carefully. However, in test cases representative of the simulations analyzed in the present work, there is no evidence for the choice of integration limits, or the assumption about the prior probability, causing any problems. The accuracy of the method for a reasonable selection of input parameters is demonstrated in the Supplemental Material [59].

As well as marginalization, a second crucial feature of the global fitting method, which provides a statistical advantage over ordinary least-squares fitting of each ISF, is accumulation. By accumulation we mean that the probability function $B(\tau, \lambda, s)$ is computed for ISFs at several $\Delta \mathbf{K}$ and the outputs multiplied together to give the relative probability of obtaining all the ISFs given the model. In previous work dealing with dynamics in the single-jumps limit [38], the end result of such an analysis is a relative likelihood function for the parameters $\tau$ and $\lambda$, which was represented as a surface plot. The accumulated function we have just described simply generalizes that function of two variables to include a third variable, the survival ratio $s$.

The generalization of the previously documented analysis method [38] to include a survival ratio $s$ is in principle simply the addition of the double, triple, and quadruple jump terms in the matrix (3), as specified in Eqs. (8)-(23). However, the grid search methods that were appropriate for the study of singlejump processes become unwieldy with the addition of an extra parameter $s$, and would become increasingly infeasible with further generalization of the model. Therefore, writing the accumulated probability as $p$, we use $-\ln (p)$ as the objective function for a non-grid-based function minimization search, in practice the MATLAB routine fminsearch [70]. The typical number of iterations required to converge on the correct parameter set is generally small compared to what would be required in a grid search, illustrated by the iteration counts for the test cases reported in the Supplemental Material [59].

\section{RESULTS AND APPLICATIONS}

\section{A. Dynamics at high friction: $\mathrm{Cp} / \mathrm{Cu}(111)$}

Experimental studies of $\mathrm{Cp} / \mathrm{Cu}(111)$ with $\mathrm{HeSE}$ were a major factor in the original development of the global fitting concept used in the present work [37,38]. $\mathrm{Cp}$ is an anionic five-membered ring formed upon dissociative adsorption of 
cyclopentadiene $\left(\mathrm{C}_{5} \mathrm{H}_{6}\right)$ on $\mathrm{Cu}(111)$. It was previously argued that rotational motion would be essentially invisible to helium atom scattering methods given the high rotational symmetry of the species, and therefore both experiments and simulations have been analyzed within the framework of effective centerof-mass motion. Scan-by-scan analysis of the experimental data was sufficient to demonstrate that the diffusion dynamics do not occur on a Bravais lattice of adsorption sites [37], but the extra statistical power afforded by the global analysis allowed the nondegeneracy of the adsorption sites to be determined to high precision [38]. The friction of $\gamma=2.5 \mathrm{ps}^{-1}$ that was used to fit the experimental data predicts an absolute jump rate near the maximum of the Kramers turnover [71] and the dynamics can therefore be reasonably assumed to be dominated by single jumps [60]. The very sharply defined probability maxima of the experimental Bayesian analysis also strongly suggest that a single-jump model was suitable. Here we confirm explicitly that the friction previously inferred does give predominantly but not exclusively single jumps, but that reducing the friction slightly leads to a take-off in the proportional of multiple jumps.

Simulations were performed on the potential energy surface whose construction was described in Sec. III B, and which is visualized in the Supplemental Material [59]. A sample simulated trajectory is also shown in the Supplemental Material. To find the jump statistics, MD simulations of duration $12 \mathrm{~ns}$ were performed with a time step of $5 \mathrm{fs}$, for 200 independent particles. The ISF was calculated for $0<t<600$ ps a range of $\Delta \mathbf{K}$ from $0.1 \AA^{-1}$ along the $\mathbf{x}$ and $\mathbf{y}$ directions, and the collection of simulated ISFs was analyzed with the global fitting method (Sec. III) to extract the most likely values of $\tau, \lambda$, and $s$. The MD simulations and analysis were repeated for different values of the friction $0.5<\gamma<$ $5.0 \mathrm{ps}^{-1}$. The resulting $\lambda$ and $s$ as a function of the friction are shown in Fig. 3. As expected, increasing the friction leads to a monotonic decrease in the survival parameter $s$ representing the proportion of long jumps, while the concentration ratio $\lambda$ remains essentially constant. The behavior of the inverse mean residence time $\tau^{-1}$ is nonmonotonic, and is plotted in the Supplemental Material. The maximum rate $\tau^{-1}$ is obtained near the experimentally determined value of the friction $\gamma=$ $2.5 \mathrm{ps}^{-1}$ in agreement with previous simulation studies [71].

\section{B. Dynamics at low friction: $\mathrm{D} / \mathrm{Pd}(\mathbf{1 1 1})$}

The interaction of hydrogen with palladium (Pd) surfaces is an extremely significant topic in applied fields including catalytic hydrogenation [74], reversible hydrogen storage [75], and a range of renewable energy technologies [76]. More generally, hydrogen dynamics on close-packed metal surfaces is a widely studied example of diffusion over a honeycomb arrangement of adsorption sites, often with a focus on quantum effects at low temperatures [10,39]. The study of rapid surface diffusion on well-defined Pd surfaces has an important contribution to make to the bottom-up understanding of the hydrogen/surface interaction including both the adiabatic, average interaction, and the dissipative coupling. We explore the classical dynamics of heavy hydrogen (deuterium, D) atoms on $\operatorname{Pd}(111)$ at elevated surface temperature $T_{s}=350 \mathrm{~K}$. The influence of nuclear quantum effects on the absolute

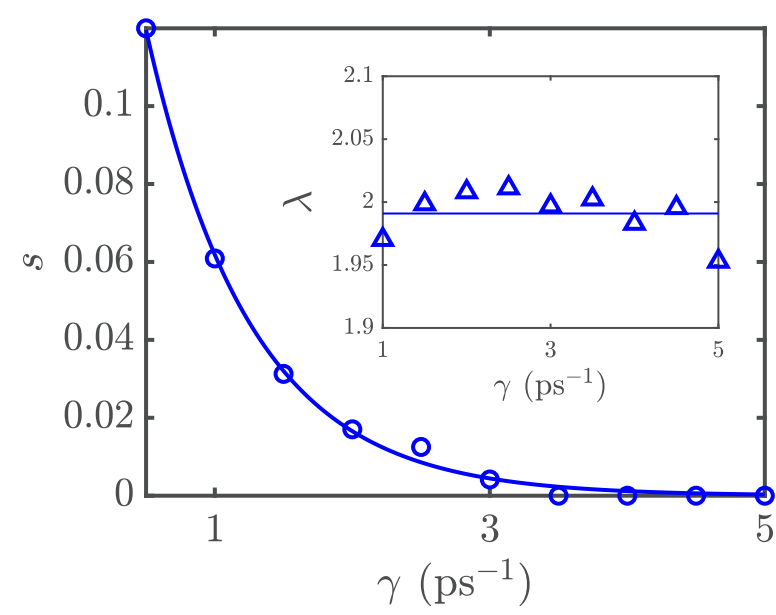

FIG. 3. Dependence of $s$ and $\lambda$ on the friction $\gamma$, for the model simulations representing center-of-mass dynamics of $\mathrm{Cp} / \mathrm{Cu}(111)$. The survival parameter $s$ exhibits a monotonic decay with increasing friction. The solid curve is an exponential fit for $s(\gamma)$, which gives a good description of the trend. The stable fitted value of $\lambda$ is shown in the inset, which confirms that despite the various assumptions in the survival ratio model (Sec. II), the model is providing a good description of the jump dynamics. The variation of the mean inverse residence time extracted from the same simulation data is expected to show a maximum $[60,72,73]$ around the experimental value of the friction $\gamma=2.5 \mathrm{ps}^{-1}$ [71], which is verified by a plot of $\tau^{-1}(\gamma)$ in the Supplemental Material [59].

jump rate and jump distribution in multidimensional quantum diffusion is not comprehensively and quantitatively settled, due to the fundamental difficulties in dynamical treatment of dissipative nonlinear quantum systems [77]. However, evidence from a range of sources suggests that the activated hopping dynamics of $\mathrm{D} / \mathrm{Pd}(111)$ at elevated temperatures can plausibly be described classically with modest corrections; a detailed discussion is given in the Supplemental Material $[31,59,60,77-83]$. Here we model the dynamics classically.

To estimate a plausible range of Langevin $\gamma$ to simulate, we can use evidence from theoretical studies of the damping effect of the free electron gas at metal surfaces. Coupling to electron-hole pair excitations on metal surfaces is a well known mechanism that gives rise to an effective Langevin friction $[16,84,85]$. One route to estimating the electronic friction is to perform a linear response analysis of the electron gas within density functional theory, which for example leads to a predicted vibrational lifetime of $\tau=0.8 \mathrm{ps}$ for a hydrogen atom adsorbed at hollow sites on $\mathrm{Cu}(111)$ [86]. In a different formulation, calculations of the electron-induced transition rates between different vibrational states of hydrogen on the $\operatorname{Pd}(111)$ surface reveal a typical transition rate of $2 \mathrm{ps}^{-1}$ [87], implying a similar lifetime on both surfaces. Vibrational spectroscopy measurements on various metal surfaces do not contradict the theoretical picture, but experimental resolution and additional broadening effects limit the measurement of very sharp vibrational lines $[88,89]$, which re-emphasises the importance of alternative measures of the friction, such as the jump distribution. Therefore we simulate the regime $0.5<$ $\gamma<5 \mathrm{ps}^{-1}$ to examine the effect of low friction $\left(\gamma \ll \omega_{0}\right)$ on the jump distribution. Although the primary function of 


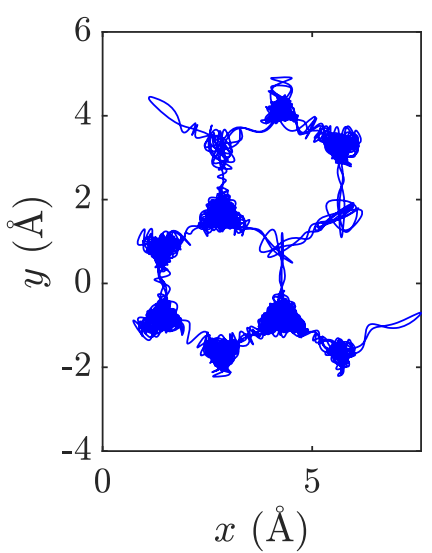

FIG. 4. Simulated 100 ps trajectory for a deuterium atom on the potential energy surface shown in Fig. 2, at a temperature $T=350 \mathrm{~K}$ and a Langevin friction $\gamma=1.0 \mathrm{ps}^{-1}$. The simulation is just long enough to show a handful of jumps. The trajectories used for the statistical analysis of hopping dynamics were considerably longer as described in the main text.

the simulations is to illustrate the application of the Bayesian fitting routine, we have given reasonable grounds to believe the simulation results have a basis in reality, and therefore have significant implications for the study of dissipation in hydrogen diffusion.

Figure 4 shows a single particle trajectory, of duration $100 \mathrm{ps}$, simulated on the PES of Fig. 2 using the LE with $\gamma=1.0 \mathrm{ps}^{-1}$, at a simulation temperature of $350 \mathrm{~K}$. The time step is $0.2 \mathrm{fs}$, which is substantially smaller than any other timescale of the simulation and correctly maintains the target simulation temperature.

ISFs were calculated at $\Delta K=\{0.1,0.2, \ldots, 3.5\} \AA^{-1}$ from simulations lasting $1.2 \mathrm{~ns}$ for 200 independent particles, and truncated at a correlation time of $60 \mathrm{ps}$. The truncation in time is a realistic feature of the HeSE experiment where only

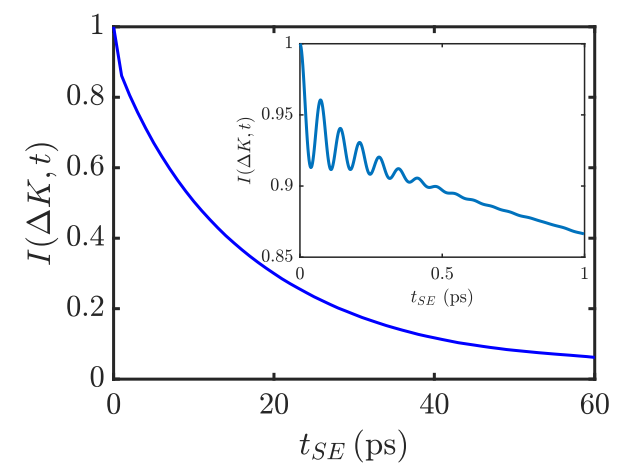

FIG. 5. ISF derived from simulated trajectories. The simulation conditions were the same as used to produce the trajectory illustrated in Fig. 4, i.e., $T=350 \mathrm{~K}$ and $\gamma=1.0 \mathrm{ps}^{-1}$. However, the total simulation time is now $1.2 \mathrm{~ns}$, and the results are averaged over 200 independent particles. The momentum transfer is $\Delta K=1.5 \AA^{-1}$, directed along the $\mathbf{x}$ direction. In the inset, the short time behavior of the ISF is shown at the time resolution of the simulations. The ISF exhibits complete vibrational dephasing over the timescale $1 / \gamma$, and matches on to the monotonic decay at longer times. a finite spin echo time is available. Figure 5 shows an ISF at $\Delta K=1.5 \AA^{-1}$, derived from a set of simulated trajectories at temperature $T_{s}=350 \mathrm{~K}$, with $\gamma=1.0 \mathrm{ps}^{-1}$. The momentum transfer is directed along the $\mathbf{x}$ direction. The ISF undergoes a nonbiexponential decay at short correlation times, before matching on to a biexponential decay (with an amplitude less than unity) at long times. The nonbiexponential decay is caused by features of the dynamics in the continuous potential that are not captured by a hopping model, such as intracell diffusion and the finite time taken to cross the diffusion barrier.

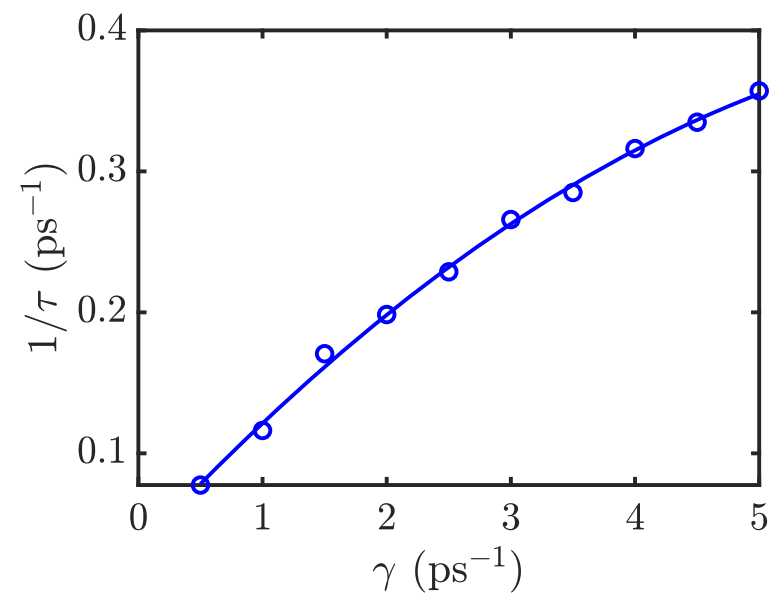

(a) Inverse residence time

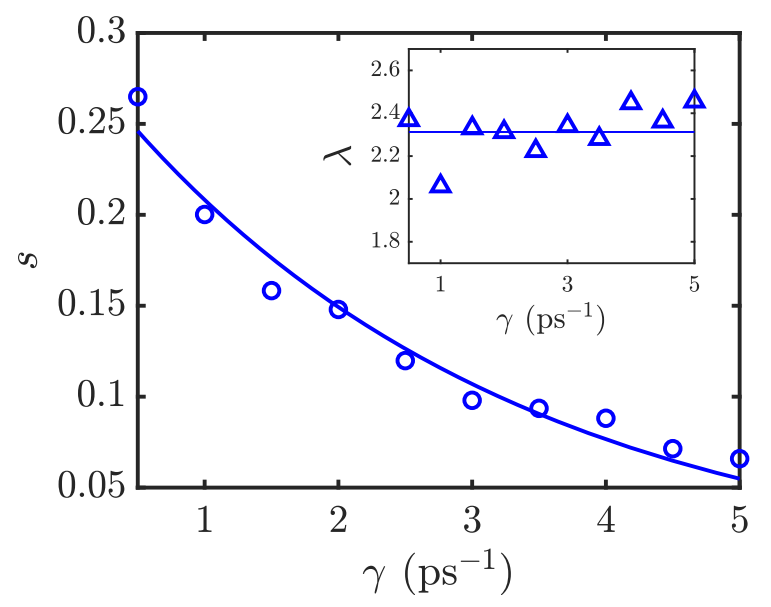

(a) Survival ratio

FIG. 6. Dependence of $\tau, s$, and $\lambda$ on the friction $\gamma$, for the Langevin simulations of $\mathrm{D} / \mathrm{Pd}(111)$. The inverse mean residence time $1 / \tau$ is shown in (a). $1 / \tau$ increases approximately linearly with $\gamma$ at the lowest $\gamma$, as expected theoretically. The solid curve is a guide to the eye. The survival ratio $s(\gamma)$ is a monotonically decreasing function of $\gamma$. The solid curve is an exponential decay, which again provides a reasonable description of the dependence of $s$ on $\gamma$. The stable fitted value of $\lambda$, shown in the inset, confirms that the survival model introduced in Sec. II provides a good description of the jump dynamics in the Langevin simulations, despite the inherent approximations. At $\gamma=1$, a reasonable estimate of electronic friction as outlined in the main text, multiple jumps are extremely prevalent which suggests that even in the presence of quantum corrections a significant proportion of multiple jumps is likely to occur in the physical system at elevated temperatures. 
The inset shows the detail of the fast initial loss of correlation including oscillations due to frustrated translational motion in the adsorption well. However, in the main plot the fast initial drop occurs over the space of one point.

Simulations were performed at different friction strength, $0.5<\gamma<5.0 \mathrm{ps}^{-1}$. For each value of $\gamma$, the global fitting method described in Sec. IIID was applied to determine the most likely set of $\tau, \lambda$, and $s$. The best fit parameters are shown as a function of $\gamma$ in Fig. 6. Because the friction is substantially lower than the frustrated translation frequency, the jump rate increases approximately linearly with the friction, in accordance with Kramers' well-known analytical result $[60,72]$. Additionally, at the lower end of the friction range, we can see that there is a high proportion of multiple jumps. Despite the approximation of completely classical dynamics, the result is highly significant for studies of hydrogen diffusion, whether with helium scattering or any other method capable of operating in a regime where multiple jumps are a strong possibility. Our numerical results make a dual contribution. First, we have established a detailed global fitting framework for inferring long jumps from ISFs and therefore HeSE data. Second, we have gathered theoretical and experimental evidence suggesting that hydrogen diffusion takes place in a low friction regime $\gamma \ll \omega_{0}$, and used Langevin simulations on a realistic model potential to confirmed the associated high proportion of long jumps in the classical limit.

\section{CONCLUSIONS}

We have derived new analytical expressions for the intermediate scattering function of an adsorbate in a jump diffusion model. In the model, the adsorbate performs long jumps on a honeycomb arrangement of adsorption sites, including jumps up to fourth-nearest neighbor in length. The model is relevant for adsorbates diffusing between hollow sites on close-packed surfaces as exemplified by the (111) faces of fcc metals. A survival-ratio model of the jump distribution was introduced in order to characterize the proportion of long jumps with a single parameter. The analytical scattering functions have been verified with kinetic Monte Carlo simulations, which define the survival ratio via the rates of allowed jumps in a process table. The analytical model, and the previously published Bayesian analysis concept, have been synthesised into a global marginalized fitting routine capable of inferring the jump distribution from simulations.

Classical molecular dynamics simulations in the Langevin framework have been carried out, using model potentials representing the diffusion of cyclopentadienyl $(\mathrm{Cp})$ anions on $\mathrm{Cu}(111)$, and deuterium atoms on $\mathrm{Pd}(111)$. The molecular dynamics simulations have been analyzed via ISFs and the global fitting framework to extract jump distributions for both systems. The results for $\mathrm{Cp} / \mathrm{Cu}(111)$ confirm the dominance of single jumps, which was assumed but not explicitly analyzed in previous studies. For $\mathrm{D} / \mathrm{Pd}(111)$ we find that at an elevated surface temperature $\left(T_{s}=350 \mathrm{~K}\right)$ and for theoretically realistic estimates of the Langevin friction based on the electron-hole pair mechanism, the jump distribution contains a significant fraction of long jumps fitted with a survival probability of approximately $20 \%$ at $\gamma=1.0 \mathrm{ps}^{-1}$. Inferring the presence of multiple jumps in experimental helium-scattering studies of hydrogen diffusion, is therefore highly feasible and would verify the low-friction regime experimentally.

\section{ACKNOWLEDGMENTS}

P.T. acknowledges the Engineering and Physical Sciences Research Council (EPSRC) for doctoral funding under award number 1363145. N.A. acknowledges a Herschel Smith fellowship. The authors thank Dr. John Ellis for helpful discussions.
[1] M. Halik, H. Klauk, U. Zschieschang, G. Schmid, C. Dehm, M. Schütz, S. Maisch, F. Effenberger, M. Brunnbauer, and F. Stellacci, Nature (London) 431, 963 (2004).

[2] P. Sutter, J.-I. Flege, and E. Sutter, Nat. Mater. 7, 406 (2008).

[3] S. Hofmann, G. Csányi, A. C. Ferrari, M. C. Payne, and J. Robertson, Phys. Rev. Lett. 95, 036101 (2005).

[4] G. Pawin, L. Wong, K.-Y. Kwon, and L. Bartels, Science 313, 961 (2006).

[5] A. K. Engstfeld, H. E. Hoster, R. J. Behm, L. D. Roelofs, X. Liu, C. Z. Wang, Y. Han, and J. W. Evans, Phys. Rev. B 86, 085442 (2012).

[6] S. Renisch, R. Schuster, J. Wintterlin, and G. Ertl, Phys. Rev. Lett. 82, 3839 (1999).

[7] P. R. Kole, H. Hedgeland, A. P. Jardine, W. Allison, J. Ellis, and G. Alexandrowicz, J. Phys.: Condens. Mat. 24, 104016 (2012).

[8] S. Paterson, W. Allison, H. Hedgeland, J. Ellis, and A. P. Jardine, Phys. Rev. Lett. 106, 256101 (2011).

[9] L. J. Lauhon and W. Ho, Phys. Rev. Lett. 85, 4566 (2000).

[10] E. McIntosh, K. Wikfeldt, J. Ellis, A. Michaelides, and W. Allison, J. Phys. Chem. Lett. 4, 1565 (2013).
[11] P. J. Feibelman, B. Hammer, J. Nørskov, F. Wagner, M. Scheffler, R. Stumpf, R. Watwe, and J. Dumesic, J. Phys. Chem. B 105, 4018 (2001).

[12] A. Tkatchenko, L. Romaner, O. T. Hofmann, E. Zojer, C. Ambrosch-Draxl, and M. Scheffler, MRS Bull. 35, 435 (2010).

[13] N. Avidor, H. Hedgeland, G. Held, A. P. Jardine, W. Allison, J. Ellis, T. Kravchuk, and G. Alexandrowicz, J. Phys. Chem. A 115, 7205 (2011).

[14] S. Baldanza, A. Cornish, R. E. Nicklin, Z. V. Zheleva, and G. Held, Surf. Sci. 629, 114 (2014).

[15] T. Mitsui, M. Rose, E. Fomin, D. Ogletree, and M. Salmerón, Surf. Sci. 540, 5 (2003).

[16] S. P. Rittmeyer, D. J. Ward, P. Gütlein, J. Ellis, W. Allison, and K. Reuter, Phys. Rev. Lett. 117, 196001 (2016).

[17] K. L. Kostov, W. Widdra, and D. Menzel, J. Phys. Chem. B 108, 14324 (2004).

[18] G. Pauer and A. Winkler, J. Chem. Phys. 120, 3864 (2004).

[19] M. J. Allen, V. C. Tung, and R. B. Kaner, Chem. Rev. 110, 132 (2010). 
[20] K. Zhang, Y. Feng, F. Wang, Z. Yang, and J. Wang, J. Mater. Chem. C 5, 11992 (2017).

[21] J. Grant and T. Haas, Surf. Sci. 21, 76 (1970).

[22] T. Land, T. Michely, R. J. Behm, J. Hemminger, and G. Comsa, Surf. Sci. 264, 261 (1992).

[23] K. M. Reddy, A. D. Gledhill, C.-H. Chen, J. M. Drexler, and N. P. Padture, Appl. Phys. Lett. 98, 113117 (2011).

[24] R. Addou, A. Dahal, P. Sutter, and M. Batzill, Appl. Phys. Lett. 100, 021601 (2012).

[25] S. H. Mok, A. Ebnonnasir, Y. Murata, S. Nie, F. K. McCarty, V. C. Ciobanu, and S. Kodambaka, Appl. Phys. Lett. 104, 101606 (2014).

[26] J. M. Cohen and A. F. Voter, J. Chem. Phys. 91, 5082 (1989).

[27] D. E. Sanders and A. E. DePristo, Surf. Sci. 264, L169 (1992).

[28] G. Boisvert and L. J. Lewis, Phys. Rev. B 54, 2880 (1996).

[29] J. Ferrón, R. Miranda, and J. J. de Miguel, Phys. Rev. B 79, 245407 (2009).

[30] T. Ala-Nissila, R. Ferrando, and S. Ying, Adv. Phys. 51, 949 (2002).

[31] L. Kristinsdóttir and E. Skúlason, Surf. Sci. 606, 1400 (2012).

[32] H. Chen, W. Zhu, and Z. Zhang, Phys. Rev. Lett. 104, 186101 (2010).

[33] F. Cinquini, F. Delbecq, and P. Sautet, Phys. Chem. Chem. Phys. 11, 11546 (2009).

[34] J. Li, E. Croiset, and L. Ricardez-Sandoval, Phys. Chem. Chem. Phys. 16, 2954 (2014).

[35] C. Stampfl and M. Scheffler, Phys. Rev. B 54, 2868 (1996).

[36] D. J. Ward, Ph.D. thesis, University of Cambridge, 2013.

[37] H. Hedgeland, B. A. J. Lechner, F. E. Tuddenham, A. P. Jardine, W. Allison, J. Ellis, M. Sacchi, S. J. Jenkins, and B. J. Hinch, Phys. Rev. Lett. 106, 186101 (2011).

[38] B. A. J. Lechner, P. R. Kole, H. Hedgeland, A. P. Jardine, W. Allison, B. J. Hinch, and J. Ellis, Phys. Rev. B 89, 121405 (2014).

[39] A. Jewell, G. Peng, M. Mattera, E. Lewis, C. Murphy, G. Kyriakou, M. Mavrikakis, and E. H. Sykes, ACS Nano 6, 10115 (2012).

[40] A. P. Jardine, G. Alexandrowicz, H. Hedgeland, W. Allison, and J. Ellis, Phys. Chem. Chem. Phys. 11, 3355 (2009).

[41] O. M. Braun and R. Ferrando, Phys. Rev. E 65, 061107 (2002).

[42] A. P. Jardine, H. Hedgeland, G. Alexandrowicz, W. Allison, and J. Ellis, Progress Surf. Sci. 84, 323 (2009).

[43] L. Van Hove, Phys. Rev. 95, 249 (1954).

[44] C. T. Chudley and R. J. Elliott, Proc. Phys. Soc. London 77, 353 (1961).

[45] G. Alexandrowicz, P. R. Kole, E. Y. M. Lee, H. Hedgeland, R. Ferrando, A. P. Jardine, W. Allison, and J. Ellis, J. Am. Chem. Soc. 130, 6789 (2008).

[46] G. Alexandrowicz, A. P. Jardine, P. Fouquet, S. Dworski, W. Allison, and J. Ellis, Phys. Rev. Lett. 93, 156103 (2004).

[47] J. M. Rowe, K. Sköld, H. E. Flotow, and J. Rush, J. Phys. Chem. Solids 32, 41 (1971).

[48] F. E. Tuddenham, H. Hedgeland, A. P. Jardine, B. A. J. Lechner, B. J. Hinch, and W. Allison, Surf. Sci. 604, 1459 (2010).

[49] D. Sivia and J. Skilling, Data Analysis: A Bayesian Tutorial, 2nd ed. (Oxford University Press, Oxford, 2005).

[50] S. Miret-Artés and E. Pollak, J. Phys.: Condens. Matter 17, S4133 (2005).
[51] A. P. Jardine, J. Ellis, and W. Allison, J. Chem. Phys. 120, 8724 (2004).

[52] F. E. Tuddenham, Ph.D. thesis, University of Cambridge, 2011.

[53] L. Onsager, Phys. Rev. 37, 405 (1931).

[54] H. Jónsson, Annu. Rev. Phys. Chem. 51, 623 (2000).

[55] A. Gross, Theoretical Surface Science: A Microscopic Perspective, 2nd ed. (Springer, Berlin, 2009).

[56] A. P. J. Jansen, An Introduction to Kinetic Monte Carlo Simulations of Surface Reactions, 4th ed., Lecture Notes in Physics, Vol. 856 (Springer, Berlin, 2012).

[57] M. Leetmaa and N. V. Skorodumova, Comput. Phys. Commun. 185, 2340 (2014).

[58] M. J. Hoffmann, S. Matera, and K. Reuter, Comput. Phys. Commun. 185, 2138 (2014).

[59] See Supplemental Material at http://link.aps.org/supplemental/ 10.1103/PhysRevB.99.115419 for further details on the evaluation of marginalized probabilities, molecular dynamics simulations and analysis for the $\mathrm{Cp} / \mathrm{Cu}(111)$ system, kinetic Monte Carlo simulations validating the analytical ISFs in the present work, and the significance of quantum corrections for the D/Pd(111) system.

[60] P. Hänggi, P. Talkner, and M. Borkovec, Rev. Mod. Phys. 62, 251 (1990).

[61] J. Zhu, Ph.D. thesis, University of Cambridge, 2015.

[62] L. Verlet, Phys. Rev. 159, 98 (1967).

[63] R. Kubo, Rep. Prog. Phys. 29, 255 (1966).

[64] The Mathworks Inc., csape (2018) [Online; accessed 11 July 2018].

[65] The Mathworks Inc., interp2 (2018) [Online; accessed 11 July 2018].

[66] M. Sacchi, S. J. Jenkins, H. Hedgeland, A. P. Jardine, and B. J. Hinch, J. Phys. Chem. C 115, 16134 (2011).

[67] B. A. J. Lechner, A. S. De Wijn, H. Hedgeland, A. P. Jardine, B. J. Hinch, W. Allison, and J. Ellis, J. Chem. Phys. 138, 194710 (2013).

[68] K. F. Riley, M. P. Hobson, and S. J. Bence, Mathematical Methods for Physics and Engineering, 3rd ed. (Cambridge University Press, Cambridge, 2006).

[69] P. R. Kole, Ph.D. thesis, University of Cambridge, 2011.

[70] The Mathworks Inc., fminsearch (2019) [Online; accessed 19 Jan 2019].

[71] B. A. J. Lechner, Ph.D. thesis, University of Cambridge, 2012; Studying Complex Surface Dynamical Systems Using Helium-3 Spin-Echo Spectroscopy, Springer Theses (Springer International, New York, 2014).

[72] H. Kramers, Physica 7, 284 (1940).

[73] H. Grabert, Phys. Rev. Lett. 61, 1683 (1988).

[74] R. Nie, H. Jiang, X. Lu, D. Zhou, and Q. Xia, Catal. Sci. Technol. 6, 1913 (2016).

[75] D. A. Gilbert, E. C. Burks, S. V. Ushakov, P. Abellan, I. Arslan, T. E. Felter, A. Navrotsky, and K. Liu, Chem. Mater. 29, 9814 (2017).

[76] B. D. Adams and A. Chen, Mater. Today 14, 282 (2011).

[77] U. Weiss, Quantum Dissipative Systems, 4th ed., Series in Modern Condensed Matter Physics, Vol. 13 (World Scientific, Singapore, 2012).

[78] P. G. Wolynes, Phys. Rev. Lett. 47, 968 (1981).

[79] H. Grabert, P. Olschowski, and U. Weiss, Phys. Rev. B 36, 1931 (1987). 
[80] I. Rips and E. Pollak, Phys. Rev. A 41, 5366 (1990).

[81] M. Topaler and N. Makri, J. Chem. Phys. 101, 7500 (1994).

[82] E. Hershkovitz, P. Talkner, E. Pollak, and Y. Georgievskii, Surf. Sci. 421, 73 (1999).

[83] Y. Georgievskii and E. Pollak, Phys. Rev. E 49, 5098 (1994).

[84] B. Hellsing and M. Persson, Phys. Scripta 29, 360 (1984).
[85] J. I. Juaristi, M. Alducin, R. Díez Muiño, H. F. Busnengo, and A. Salin, Phys. Rev. Lett. 100, 116102 (2008).

[86] J. R. Trail, D. Bird, M. Persson, and S. Holloway, J. Chem. Phys. 119, 4539 (2003).

[87] J. Tremblay, J. Chem. Phys. 138, 244106 (2013).

[88] G. Lee and E. W. Plummer, Surf. Sci. 498, 229 (2002).

[89] K. L. Kostov, W. Widdra, and D. Menzel, Surf. Sci. 560, 130 (2004). 